INPLASY

PROTOCOL

To cite: Hu et al. Adverse effects of chemotherapy with or without monoclonal antibody drug therapy for advanced esophageal cancer: a metaanalysis of randomized controlled trial. Inplasy protocol 202190100. doi:

10.37766/inplasy2021.9.0100

Received: 27 September 2021

Published: 27 September 2021

Corresponding author:

Rongqiu Hu

hurrrq@163.com

Author Affiliation:

The Southwest Medical University.

Support: Nursing research project of SC.

Review Stage at time of this submission: Preliminary searches.

Conflicts of interest: None declared.

\section{Adverse effects of chemotherapy with or without monoclonal antibody drug therapy for advanced esophageal cancer: a meta-analysis of randomized
controlled trial}

Hu, RQ1; Cheng, YL2; Tian, T3; Luo, W4; Wang, YP5.

Review question / Objective: P: Esophageal carcinoma; I: Chemotherapy combined with monoclonal antibody drugs Chemotherapy plus monoclonal antibody drugs; C: Chemotherapy; $0:$ The incidence of adverse reactions; $S$ : Randomized controlled trial.

Eligibility criteria: We used the following inclusion criteria: (1) only esophagus cancer trials have been included in the analysis, including esophageal squamous cell carcinoma (ESCS), adenocarcinoma or undifferentiated carcinoma of the esophagus, and adenocarcinoma of the thoracic esophagus. (2) Metastatic, localized esophageal cancer, esophagogastric junction cancer and advanced inoperable patients were accepted. (3) Comparing the drug-related side effects and adverse reactions of monoclonal antibody drugs with monoclonal antibody drugs plus chemotherapy. (4) Independent clinical trials with an analysis of clinical data). (5) RCTs. Exclusion criteria Our exclusion criteria included (1) Patients less than 20 subjects, (2) Reviews and qualitative studies, (3) Animal study and cell experiment and (4) Duplicated reports.(5) Results not reported exactly.

INPLASY registration number: This protocol was registered with the International Platform of Registered Systematic Review and Meta-Analysis Protocols (INPLASY) on 27 September 2021 and was last updated on 27 September 2021 (registration number INPLASY202190100).

\section{INTRODUCTION}

Review question / Objective: P: Esophageal carcinoma; I: Chemotherapy combined with monoclonal antibody drugsChemotherapy plus monoclonal antibody drugs; C: Chemotherapy; O: The incidence of adverse reactions; $S$ : Randomized controlled trial.

Rationale: Two independent reviewers extracted the data from each included article. The data extracted from the eligible 
studies were recorded as follows: author, year of publication, number of participants, state of health, time of therapy, study type, treatment protocols, disease diagnosis. Statistical analysis was performed using RevMan 5.4 software recommended by the Cochrane Library. Two-tailed $p$ values $<0.05$ were considered statistically significant. The heterogeneity between the included studies was quantified by Chi-square test and I-square test and was considered to be obvious if $p$ was $50 \%$. The ratio of the number of people with adverse reactions to the number of people without adverse reactions in the case group divided by the ratio of the number of people with adverse reactions to the number of people without adverse reactions in the control group. When $p$ was $50 \%$ (high heterogeneity), random-effects models were applied. When $p$ was $>0.10$ and 12 was $<50 \%$ (low heterogeneity), fixed-effects models were used.

Condition being studied: Esophageal cancer is a highly lethal disease, the sixth leading cause of cancer-related mortality in the world(PMID: 31798770) and the eighth most common cancer, with a 5-year survival rate of $15-25 \%$, affecting more than 450,000 people worldwide(PMID: 34056697). Treatment methods include surgical resection, chemotherapy, radiotherapy, targeted therapy, and immunotherapy. For early esophageal cancer, surgical resection is the radical treatment(PMID: 34494553). However, due to late symptoms and diagnosis, the prognosis of most patients is poor. Therefore, chemotherapy has been the main means of treatment for advanced esophageal cancer(PMID: 34056697). For locally advanced esophageal cancer, nearly half of patients are not suitable for surgery, and a combination of neoadjuvant chemoradiotherapy and surgery usually achieves the best treatment outcome(PMID: 34278011). Neoadjuvant therapy (including chemotherapy plus radiotherapy and/or immunotherapy) can effectively treat esophageal cancer and even achieve pathological complete response(PMID: 33644048). In recent years, epidermal growth factor receptor (EGFR) has been found to play an indispensable role in esophageal tumorigenesis(PMID: 33105560). Abnormal activation of EGFR by amplification, overexpression, or mutation can induce tumor cell proliferation, migration, and metastasis(PMID:15120370). A variety of monoclonal antibody inhibitors, including cetuximab, nittuzumab and panizumab, bind to the extracellular domain of EGFR and compete with endogenous ligand to block ligand-binding region and ligand-induced EGFR tyrosine kinase activation(PMID: 33753446), thereby delaying or even treating esophageal cancer. However, for advanced esophageal cancer, chemoradiotherapy or monoclonal antibody inhibitors combined with chemoradiotherapy will have varying degrees of adverse reactions. It mainly includes gastrointestinal adverse reactions, hematological adverse reactions, skin and nervous system adverse reactions, etc. (PMID: 27863481), presenting with nausea, vomiting, diarrhea, anorexia, hair loss, rash, peripheral neuropathy, leukopenia, neutropenia, thrombocytopenia, and hypokalemia and hypomagnesia (PMID:34121703). This meta-analysis mainly evaluated whether the incidence of adverse reactions in patients with advanced esophageal cancer was improved by using monoclonal antibody inhibitors compared with monoclonal antibody inhibitors combined with chemoradiotherapy, thus providing some guidance for the selection of clinical treatment strategies for patients with advanced esophageal cancer.

\section{METHODS}

Search strategy: The literature search was conducted using Cochrane Library, the PubMed database, the Embase database, the China National Knowledge Infrastructure database, the Wanfang database, the VIP database.

Participant or population: Patients with esophageal carcinoma.

Intervention: Chemotherapy with monoclonal antibody drug therapy for advanced esophageal carcinoma cancer. 


\section{Comparator: Chemotherapy.}

Study designs to be included: Randomized controlled trial.

Eligibility criteria: We used the following inclusion criteria: (1) only esophagus cancer trials have been included in the analysis, including esophageal squamous cell carcinoma (ESCS), adenocarcinoma or undifferentiated carcinoma of the esophagus, and adenocarcinoma of the thoracic esophagus. (2) Metastatic, localized esophageal cancer, esophagogastric junction cancer and advanced inoperable patients were accepted. (3) Comparing the drug-related side effects and adverse reactions of monoclonal antibody drugs with monoclonal antibody drugs plus chemotherapy. (4) Independent clinical trials with an analysis of clinical data). (5) RCTs. Exclusion criteria Our exclusion criteria included (1) Patients less than 20 subjects, (2) Reviews and qualitative studies, (3) Animal study and cell experiment and (4) Duplicated reports.(5) Results not reported exactly.

Information sources: Cochrane Library, the PubMed database, the Embase database, the China National Knowledge Infrastructure database, the Wanfang database, the VIP database.

Main outcome(s): Evaluated the advanced esophageal cancer chemotherapy plus chemotherapy alone single resistance and the differences of two treatments of adverse events, including peripheral neuropathy, neutrophils, white blood cells, anemia, platelets, pneumonia, diarrhea, nausea, anorexia, vomiting, rash, hair loss, fever, febrile neutropenia, infections, allergies, low potassium, magnesium, fatigue.

Data management: Two independent reviewers extracted the data from each included article. The data extracted from the eligible studies were recorded as follows: author, year of publication, number of participants, state of health, time of therapy, study type, treatment protocols, disease diagnosis. Statistical analysis was performed using RevMan 5.3 software recommended by the Cochrane Library. Two-tailed $p$ values $<0.05$ were considered statistically significant.The heterogeneity between the included studies was quantified by Chi-square test and I-square test and was considered to be obvious if $p$ was $50 \%$. The ratio of the number of people with adverse reactions to the number of people without adverse reactions in the case group divided by the ratio of the number of people with adverse reactions to the number of people without adverse reactions in the control group. When $p$ was $50 \%$ (high heterogeneity), random-effects models were applied. When p was $>0.10$ and 12 was $<50 \%$ (low heterogeneity), fixed-effects models were used.

Quality assessment / Risk of bias analysis: Cochrane Collaboration network bias risk assessment tool.

Strategy of data synthesis: Two-tailed p values $<0.05$ were considered statistically significant.The heterogeneity between the included studies was quantified by Chisquare test and I-square test and was considered to be obvious if $p$ was $\mathbf{5 0} \%$. The ratio of the number of people with adverse reactions to the number of people without adverse reactions in the case group divided by the ratio of the number of people with adverse reactions to the number of people without adverse reactions in the control group. When p was $50 \%$ (high heterogeneity), random-effects models were applied. When $p$ was $>0.10$ and $\mathrm{I} 2$ was $<50 \%$ (low heterogeneity), fixed-effects models were used.

Subgroup analysis: For indicators with high heterogeneity, subgroup analysis was performed one by one to check the source of heterogeneity.

Sensitivity analysis: For indicators with high heterogeneity, sensitivity analysis was conducted one by one to check the source of heterogeneity.

Language: English. 
Country(ies) involved: China.

Other relevant information: Relying on the Affiliated Hospital of Southwest Medical University and Affiliated Hospital of Traditional Chinese Medicine in Luzhou city, Sichuan Province, China, our team has a sound medical system and is able to complete this study.

Keywords: Esophageal Neoplasms; Chemotherapy; Antibodies, Monoclonal, Humanized; Drug-Related Side Effects and Adverse Reactions; Meta.

Contributions of each author:

Author 1 - Rongqiu $\mathrm{Hu}$ - The author screened literature, collected data and drafted the manuscript.

Email: hurrrq@163.com

Author 2 - Yonglang Cheng - The author provided statistical expertise and drafted the manuscript.

Email: chengyonglang666@163.com

Author 3 - Tian Tian - The author contributed to the development of the selection criteria, and the risk of bias assessment strategy.

Author 4 - Wen Luo.

Author 5 - Yiping Wang - Selected topic and instructor.

Email: wangyiping526@163.com 